Please do not remove this page

RMIT

UNIVERSITY

\title{
Enhanced quantum efficiency from a mosaic of two dimensional MoS2 formed onto aminosilane functionalised substrates
}

Wang, Yichao; Della Gaspera, Enrico; Carey, Benjamin; Atkin, Paul; Berean, Kyle; Clark, Rhiannon; Cole, Ivan

https://researchrepository.rmit.edu.au/esploro/outputs/9921860154601341/filesAndLinks?institution=61RMIT_INST\&index=null

Wang, Y., Della Gaspera, E., Carey, B., Atkin, P., Berean, K., Clark, R., Cole, I., Xu, Z.-Q., Zhang, Y., Bao, Q., Ou, J., Daeneke, T., \& Kalantar Zadeh, K. (2016). Enhanced quantum efficiency from a mosaic of two dimensional MoS2 formed onto aminosilane functionalised substrates. Nanoscale, 8(24), 12258-12266. https://doi.org/10.1039/c6nr02197b

Document Version: Accepted Manuscript

Published Version: https://doi.org/10.1039/c6nr02197b 
Thank you for downloading this document from the RMIT Research Repository.

The RMIT Research Repository is an open access database showcasing the research outputs of RMIT University researchers.

RMIT Research Repository: http://researchbank.rmit.edu.aul

Citation:
Wang, Y, Della Gaspera, E, Carey, B, Atkin, P, Berean, K, Clark, R, Cole, I, Xu, Z,
Zhang, Y, Bao, Q, Ou, J, Daeneke, T and Kalantar Zadeh, K 2016, 'Enhanced
quantum efficiency from a mosaic of two dimensional MoS2 formed onto
aminosilane functionalised substrates', Nanoscale, vol. 8, no. 24, pp. 12258-12266.

See this record in the RMIT Research Repository at:

https://researchbank.rmit.edu.au/view/rmit:37879

Version: Accepted Manuscript

\section{Copyright Statement:}

(C) This journal is (c) The Royal Society of Chemistry 2016

Link to Published Version:

https://dx.doi.org/10.1039/c6nr02197b 


\title{
Enhanced quantum efficiency from a mosaic of two dimensional $\mathrm{MoS}_{2}$ formed onto aminosilane functionalised substrates
}

Received 00th January 20xx, Accepted 00th January 20xx

DOI: $10.1039 / \times 0 x \times 00000 x$

\section{www.rsc.org/}

\author{
Yichao Wang, ${ }^{a}$ Enrico Della Gaspera, ${ }^{b, c}$ Benjamin J. Carey, ${ }^{a}$ Paul Atkin, ${ }^{a, c}$ Kyle J. Berean, ${ }^{a}$ Rhiannon \\ M. Clark, ${ }^{a, c}$ Ivan S. Cole, ${ }^{c}$ Zai-Quan Xu, ${ }^{d}$ Yupeng Zhang, ${ }^{d}$ Qiaoliang Bao, ${ }^{d}$ Jian Zhen Ou, ${ }^{a}$ Torben \\ Daeneke, ${ }^{a}$ and Kourosh Kalantar-zadeh ${ }^{a}$
}

\begin{abstract}
Developing scalable methods of growing two dimensional molybdenum disulphide (2D MoS $)_{2}$ of strong optical properties, on any desired substrates, is a necessary step to move towards industrial uptake of this material for optical applications. In this study, $\mathrm{Si} / \mathrm{SiO}_{2}$ substrates were functionalised using self-assembled monolayers of three different aminosilanes with various number of amine groups and molecular lengths, as underlayers for enhancing the adherence of molybdenum precursor. The tetrahedral $\left[\mathrm{MoS}_{4}\right]^{2-}$ anion groups from the molybdenum precursor were bonded on these silanised $\mathrm{Si} / \mathrm{SiO}_{2}$ substrates afterwards. The substrates were then treated with a combined thermolysis and sulphurisation step. The results showed that silanisation of the substrates using longest chains and largest number of amine groups provided a good foundation to grow quasi 2D MoS 2 made from adjacent flakes in a mosaic formation. Microscopic and spectroscopic investigations revealed that these quasi 2D MoS formed using this long chain aminosilane resulted in flakes with lateral dimensions in micron and submicron ranges composed of adjoining $\mathrm{MoS}_{2}$ pieces of 20 to $60 \mathrm{~nm}$ in lateral dimensions, dominantly made of 3 to $5 \mathrm{MoS}_{2}$ fundamental layers. The obtained quasi 2D MoS shows a high internal quantum efficiency of $2.6 \%$ associateed to the quantum confinement effect and high stoichiometry of the adjoining nanoflakes that form the structure of the sheets. The synthesis technique in this study is reliable, facile and offers a procedure to form large, scaleable and patternable quasi $2 \mathrm{D} \mathrm{MoS}_{2}$ sheets on various substrates with enhanced optical properties for practical applications.
\end{abstract}

\section{Introduction}

Two-dimensional (2D) transition metal dichalcogenides (TMDs), and particularly 2D molybdenum disulphide $\left(\mathrm{MoS}_{2}\right)$, have attracted great attention in recent years, owing to their unique electronic and optical properties. ${ }^{1-3}$

In comparison to its bulk counterpart, which has an indirect band gap of $\sim 1.3 \mathrm{eV},{ }^{4}$ monolayered $\mathrm{MoS}_{2}$ has a direct bandgap of $\sim 1.9 \mathrm{eV} .^{4,5}$ Photoluminescence (PL) emission inherited from this direct band gap, make monolayered $\mathrm{MoS}_{2}$ suitable for many optical units. ${ }^{6}$ The high on-off current ratio and good electronic performance exhibited by monolayered $\mathrm{MoS}_{2}$ transistors suggest their promise in future electronic circuits, requiring low stand-by power attractive for the realisation of

\footnotetext{
${ }^{a .}$ School of Electrical and Computer Engineering, RMIT University, Melbourne, Victoria, Australia

${ }^{b}$ School of Science, RMIT University, Melbourne, Victoria, Australia

c. CSIRO Manufacturing Flagship, Clayton, Victoria, Australia

${ }^{d .}$ Department of Materials Science and Engineering, Faculty of Engineering, Monash University, Clayton 3800, Victoria, Australia

Email:kourosh.kalantar@rmit.edu.au, jianzhen.ou@rmit.edu.au

Electronic Supplementary Information (ESI) available: Supplementary figures. See DOI: $10.1039 / \mathrm{x} 0 \times x \times 0000 \mathrm{x}$
}

2D optoelectronic devices. ${ }^{6}$ To date, 2D $\mathrm{MoS}_{2}$ has found numerous potentials in units including those incorporated in field-effect transistors (FETs), ${ }^{7-10}$ catalysts, ${ }^{11-16}$ energy storage, ${ }^{17,18}$ photodetectors, ${ }^{19}$ gas separation membranes, ${ }^{20}$ piezoelectric systems, ${ }^{21}$ bio and gas sensors, ${ }^{1,22-26}$ and photovoltaics. ${ }^{27}$ Therefore, synthesis of wafer scale 2D MoS 2 using facile and reliable approaches is essential for widespread application of this material in optical and electronic devices.

In nature, $2 \mathrm{H}$ phase of $\mathrm{MoS}_{2}$ has a stratified crystal. Many top-down techniques such as micromechanical cleavage, ${ }^{4,7}$ liquid exfoliation ${ }^{28-31}$ and intercalation-assisted exfoliation ${ }^{32}$ have been used for obtaining atomically thin $\mathrm{MoS}_{2}$ nanoflakes. However, such exfoliation methods are impractical for wafer processes in optical and electronic industries. The yield for mechanical cleavage methods is generally low. ${ }^{33}$ Liquid exfoliation always results in laterally polydisperse samples. ${ }^{28,29}$ Many intercalation-assisted exfoliation processes involves the use of hazardous liquids such as butyllithium, which are very reactive and explosive reagents.

The bottom-up techniques are also widely investigated for growing large area $\mathrm{MoS}_{2}$ thin sheets. They generally rely on the reactions of selected molybdenum-containing compounds on the surface of substrates, together with sulphurisation and 
annealing steps. For instance, vacuum deposition of $\mathrm{Mo}^{34}$ $\mathrm{MoO}_{2}$ microcrystals, ${ }^{35}$ molybdenum trioxide $\left(\mathrm{MoO}_{3}\right)^{6,36,37}$ and $\mathrm{MoCl}_{5}$ powders, ${ }^{38,39}$ followed by sulphurisation processes have been demonstrated. These methods have shown promise in synthesising 2D MoS 2 on substrates such as sapphire, ${ }^{40}$ while monolayer of $\mathrm{MoS}_{2}$ in triangular islands of tens of micrometres in lateral dimensions can be obtained. ${ }^{6,37,41}$ Many of these reactions normally tend to partially form $\mathrm{MoS}_{2}$ nanoparticles or nanorod structures during the synthesis and significant tunings are required. ${ }^{36,42}$

To promote the formation of layered $\mathrm{MoS}_{2}$, it is common to make use of solution based precursors. ${ }^{53}$ These precursors are usually placed on the surface of the substrates using methods such as dip-coating ${ }^{40}$ and spin-coating. ${ }^{41}$ As one of the first demonstrations on these approaches, Liu et al. used a two-step thermolysis process to obtain few-layered $\mathrm{MoS}_{2}$ thin films. ${ }^{40}$ Molybdenum compound was first formed on the substrate and then converted into $2 \mathrm{D} \mathrm{MoS}_{2}$ by annealing and sulphurisation steps. Although the technique leads to high quality $\mathrm{MoS}_{2}$ thin films, the dip-coating process is not compatible with conventional semiconductor manufacturing practices due to its unsuitability for many large-area applications. ${ }^{42}$ Spin-coating is likely to perform worse as it produces uneven thicknesses of the precursor on the wafer. ${ }^{4}$

$\mathrm{SiO}_{2}$ covered substrates, such as $\mathrm{SiO}_{2} / \mathrm{Si}$, are the most important of all wafers to form 2D $\mathrm{MoS}_{2}$ onto due to their fundamental significance in electronic and optical device industries. However, so far most of the reports on 2D MoS 2 deposition on $\mathrm{SiO}_{2}$ have demonstrated lack of localised crystallinity for 2D material's domains due to the relatively high surface roughness and amorphous nature of $\mathrm{SiO}_{2}$ covered substrates. This leads to reduced optical qualities. ${ }^{43-45}$ As such, devising a method that can result in large area $2 \mathrm{D} \mathrm{MoS}_{2}$ with improved and enhanced optical properties, on $\mathrm{SiO}_{2} / \mathrm{Si}$ substrates is of great importance.

Many aspects should be considered in the synthesis of 2D $\mathrm{MoS}_{2}$ with high optical properties. One of the main issues is that 2D MoS 2 has a naturally relatively low internal quantum efficiency which makes it difficult to incorporate in many practical applications in optics. The quantum yield for prototypical 2D MoS 2 has been reported to be less than $1.0 \%,{ }^{46}$ which results in low optical intensity of light emitting diodes based this material. ${ }^{46}$ Also this low quantum yield comes as an obstacle for many bio medical applications in which the readability of the optical signal defines the minimum detection limits. ${ }^{47}$ We have previously shown that it is possible to increase the quantum yield by reducing the lateral dimension of $\mathrm{MoS}_{2}$ nanoflakes in suspensions. ${ }^{26}$ Based on this past observation, we hypothesize that if $2 \mathrm{D} \mathrm{MoS}$ laid on a substrate is made of small adjoining flakes, quantum yield will be enhanced. Creating 2D semiconductors from adjoining flakes may be disadvantageous for making electronic devices but can be of great benefit for developing enhanced optical unit.

One of the approaches that can be potentially used for preparing the surface of substrates for precursors is the use of silane compounds. Silanisation is one of the most used methods for the functionalisation of $\mathrm{SiO}_{2}$ surface. ${ }^{48-50}$

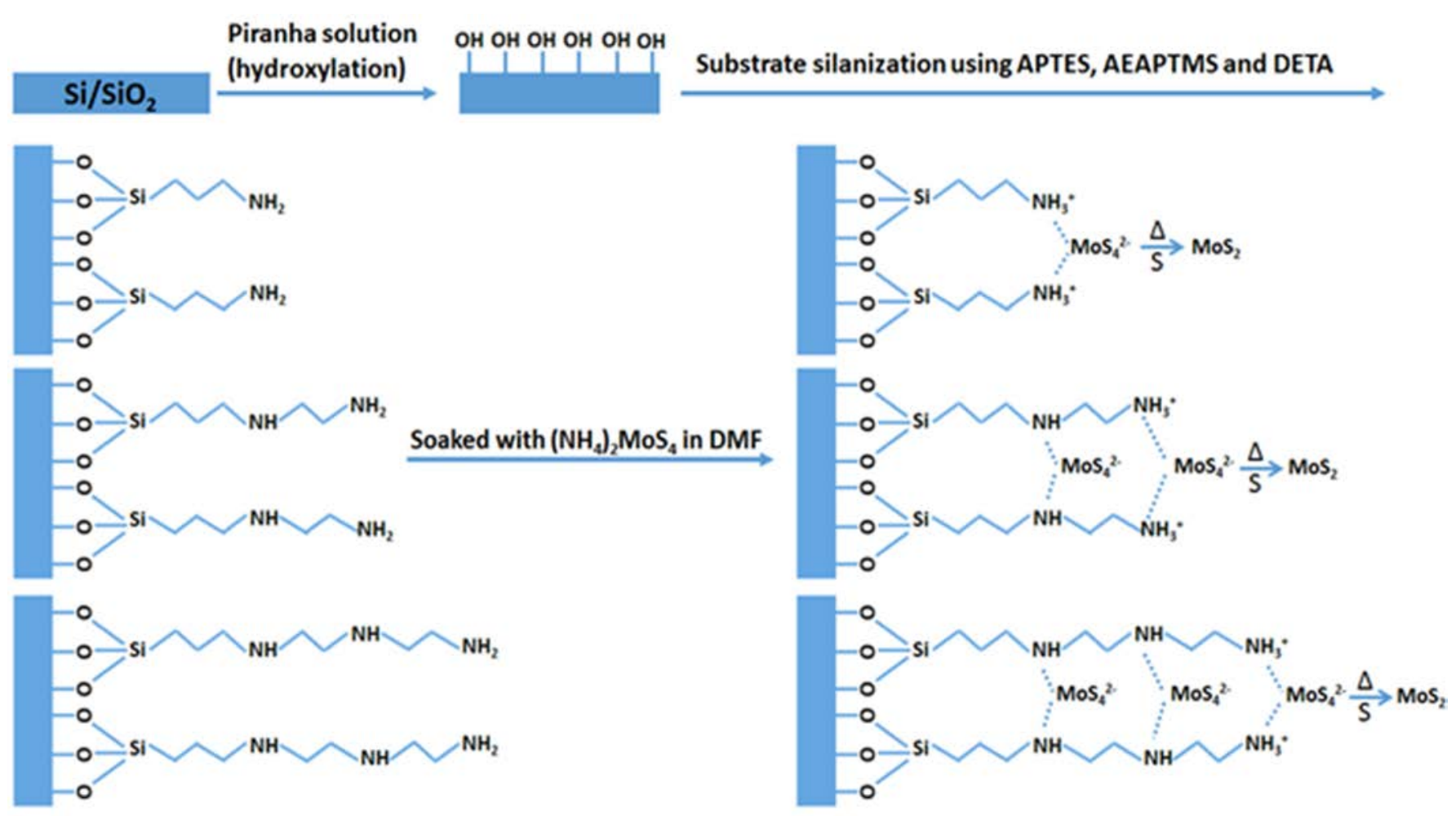

\section{$\underset{\mathrm{S}}{\vec{\Delta}}$ : Thermolysis and sulphurisation}

Scheme $1 \mathrm{Si} / \mathrm{SiO}_{2}$ substrates are cleaned and activated in a piranha solution and modified with APTES, AEAPTMS and DETA. Tetrahedral $\left[\mathrm{MoS}_{4}\right]^{2-}$ anion group is bonded on the aminosilane functionalised substrates (only idealised coverage has been shown), after that the substrates are treated through a combined thermolysis and sulphurisation process. 
However, the applicability of silanes has not been fully investigated in the deposition of $2 \mathrm{D} \mathrm{MoS}_{2}$ or any other TMDs. Specifically, we are curious to investigate whether the concept of quasi 2D MoS${ }_{2}$, with enhanced optical properties, can be realized using silane based growth. Therefore in this study, we demonstrate the formation of quasi 2D $\mathrm{MoS}_{2}$, made of adjoining flakes, on silanised $\mathrm{Si} / \mathrm{SiO}_{2}$ substrates using a combined thermolysis and sulphurisation of the attached $\left[\mathrm{MoS}_{4}\right]^{2-}$ anion groups. Three different self-assembled monolayers of aminosilane groups of - (3Aminopropyl)triethoxysilane (APTES), $\mathrm{N}$-[3(Trimethoxysilyl)propyl]ethylenediamine (AEAPTMS) and $N^{1}-(3-$ Trimethoxysilylpropyl)diethylenetriamine (DETA) are used. These groups have different molecular lengths and amine groups on their backbones. It is shown that DETA, with a longer chain and larger number of the amine group, is the most successful of them in establishing larger size quasi 2D $\mathrm{MoS}_{2}$ made of small flakes on $\mathrm{Si} / \mathrm{SiO}_{2}$ substrates. To assess the physicochemical properties of the synthesised quasi 2D MoS 2 , atomic force microscopy (AFM), $\mathrm{X}$-ray photoelectron spectroscopy (XPS) as well as Raman and PL mapping are conducted. The samples are assessed for their optical properties by measuring the internal quantum efficiency and PL decay dynamics.

\section{Results and discussions}

Quasi 2D $\mathrm{MoS}_{2}$ flakes were formed on $\mathrm{Si} / \mathrm{SiO}_{2}$ substrates via thermolysis and sulphurisation after the substrates are silanised using three different aminosilanes. Details are presented in the Methods section. In brief, the $\mathrm{Si} / \mathrm{SiO}_{2}$ substrates were cleaned and hydroxylated in a piranha solution. After that the wafers were immersed in freshly prepared APTES, AEAPTMS and DETA solutions in ethanol. Then the substrates were soaked in a solution-phase precursor of $\left(\mathrm{NH}_{4}\right)_{2} \mathrm{MoS}_{4}$ in dimethylformamide (DMF). In the following step, the samples were treated using a thermolysis and sulphurisation process. The synthesis process is illustrated in Scheme 1.

The interaction between amine groups of aminosilane and $\left(\mathrm{NH}_{4}\right)_{2} \mathrm{MoS}_{4}$ is described by:

$$
2\left(\mathrm{R}-\mathrm{NH}_{2}\right)+\left(\mathrm{NH}_{4}\right)_{2} \mathrm{MoS}_{4} \rightarrow 2\left(\mathrm{R}-\mathrm{NH}_{3}\right) \mathrm{MoS}_{4}+2 \mathrm{NH}_{3} \uparrow
$$

in which $\mathrm{R}$ is all the structure of aminosilane without one $\mathrm{NH}_{2}$ functional group. The formula is based on the acid-base reaction between aminosilanes and $\left(\mathrm{NH}_{4}\right)_{2} \mathrm{MoS}_{4}$. Here, $\mathrm{NH}_{3}$ as a weaker base can be released and was removed in the vacuum oven. This leads to a shift of the reaction equilibrium and tetrahedral $\left[\mathrm{MoS}_{4}\right]^{2-}$ anion group non-reversible interaction on the substrate. The same principle has been illustrated for the reaction of $\left(\mathrm{NH}_{4}\right)_{2} \mathrm{MoS}_{4}$ with ethylenediamine to obtain the diammonium salt. ${ }^{51}$

The control of surface wettability via functional groups plays an important role in study of the surface and ion exchange properties. ${ }^{52}$ As a result, water contact angle (WCA) measurements were conducted on the piranha treated and three different silanised $\mathrm{Si} / \mathrm{SiO}_{2}$ substrates. The piranha treated $\mathrm{Si} / \mathrm{SiO}_{2}$ substrate shows strong hydrophilicity (Fig. 1a), resulting in a WCA value of $23.5^{\circ} \pm 0.8^{\circ}$. This is due to the appearance of $\mathrm{OH}$ group on the surface. After treated with the piranha solution, the cross-linked Si$\mathrm{O}$ bond on $\mathrm{SiO}_{2}$ surface were oxidised and hydroxylated by hydrogen peroxide $\left(\mathrm{H}_{2} \mathrm{O}_{2}\right)$ and ammonium hydroxide $\left(\mathrm{NH}_{4} \mathrm{OH}\right)$. After the silanisation, aminosilanes of APTES, AEAPTMS and DETA in this

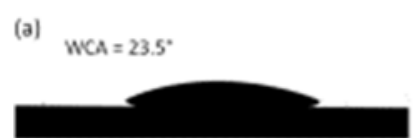

(b)
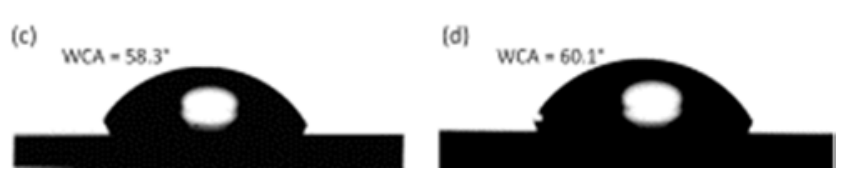

Fig. 1 WCA measurements performed on (a) piranha treated $\mathrm{Si} / \mathrm{SiO}_{2}$ substrate and on $\mathrm{Si} / \mathrm{SiO}_{2}$ substrates modified with: (b) APTES, (c) AEAPTMS, and (d) DETA.

study, with their alky chains, ethoxyl and amine groups appear on the surface. In these cases, the WCA increased to $52.8^{\circ} \pm 0.5^{\circ}, 58.3$ ${ }^{\circ} \pm 0.2^{\circ}$ and $60.1^{\circ} \pm 0.8^{\circ}$, for APTES, AEAPTMS and DETA, respectively (Fig. $1 \mathrm{~b}-\mathrm{d}$ ). This corresponds to an increasingly higher WCA and reduction in the degree of hydrophilicity, indicating that the longer DETA chain resulted in a larger contact angle. The larger contact angle is likely to promote the precursor reaction on the surface of the substrate, which is beneficial for obtaining larger size quasi 2D MoS flakes.

Silanisation process provides amine groups on $\mathrm{Si} / \mathrm{SiO}_{2}$ surface which facilitates the $\left[\mathrm{MoS}_{4}\right]^{2-}$ anion interaction. After the substrates were treated using thermolysis and sulphurisation, quasi 2D MoS 2 nanoflakes were formed. AFM was used for characterising the surface morphology and thickness of quasi 2D MoS . The topographic images and corresponding height profiles for the obtained flakes are presented in Fig. 2a-c.

As can be seen from Fig. 2a, the nanoflakes synthesised on APTES silanised substrates have lateral dimensions in the order of $\sim 100 \mathrm{~nm}$ and are sparsely located on the substrate. Clear steps of $\sim 3-4 \mathrm{~nm}$ (corresponding to 4-6 monolayers of $\mathrm{MoS}_{2}$ - thickness of monolayer $\mathrm{MoS}_{2}$ is $0.65 \mathrm{~nm}$ ) can be observed in typical nanoflakes. For the $\mathrm{MoS}_{2}$ grown on an AEAPTMS silanised substrate, larger flakes begin to appear. The lateral dimensions are increased to $\sim 350 \mathrm{~nm}$ and thicknesses of the flakes are between 3 and $5 \mathrm{~nm}$ (Fig. $2 b)$. From the AFM images, it is revealed that the DETA silanised substrate provides the best platform to grow the largest quasi $2 \mathrm{D}$ $\mathrm{MoS}_{2}$ nanosheets. From the example presented in Fig. 2c, it is seen that the obtained nanoflakes had a thickness of $\sim 2-3 \mathrm{~nm}$, which corresponds to $\sim 3$ to $5 \mathrm{MoS}_{2}$ monolayers. DETA has more bonding sites which has larger number of $-\mathrm{NH}_{2}$ groups. It was also shown that DETA makes the largest WCA. As a result, $\left[\mathrm{MoS}_{4}\right]^{2-}$ groups have more possible affinity sites and highest probability to come close and interact with the DETA silanised substrate (Scheme 1), compared to APTES and AEAPTMS treated surfaces, leading to a larger dimension of quasi 2D MoS 2 nanoflakes. In our large samples, the surface roughness $(\sigma)$ was measured using AFM. The surface roughness of the bare $\mathrm{Si} / \mathrm{SiO}_{2}$, DETA silanised, and $\mathrm{MoS}_{2}$ grown on DETA silanised substrates were measured and compared with the background roughness. From the analysis, the root mean square (RMS) surface roughness for the DETA silanised sample and its background $\mathrm{SiO}_{2} / \mathrm{Si}$ substrate was found to be $\sim 400$ and $130 \mathrm{pm}$, respectively, confirming that surface functionalization led to a jagged surface and possibly made of smaller adjoining connected flakes. The other important observation is the fact that jagged 2D $\mathrm{MoS}_{2}$ formed on DETA silanised substrates are made of adjoining small flakes of $\sim 30-50 \mathrm{~nm}$ (as an example Fig. 2d). The schematic map which presents the formation of this unique $2 \mathrm{D}$ structure is 
(a)

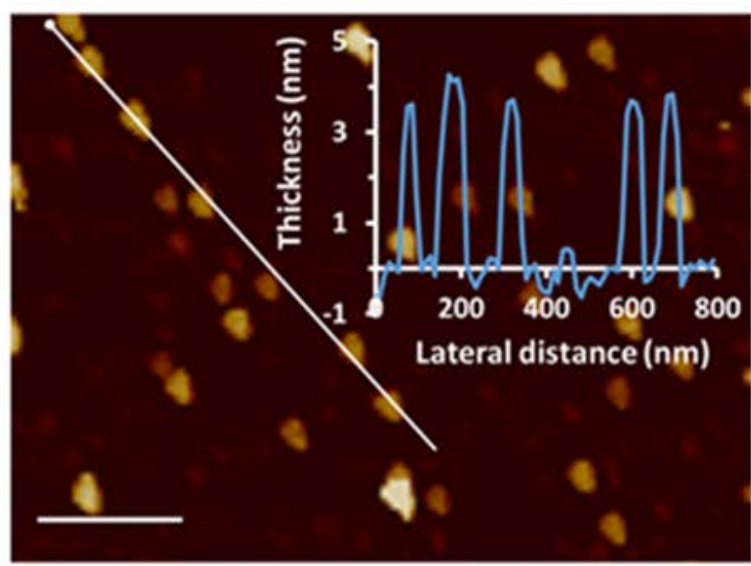

(c)

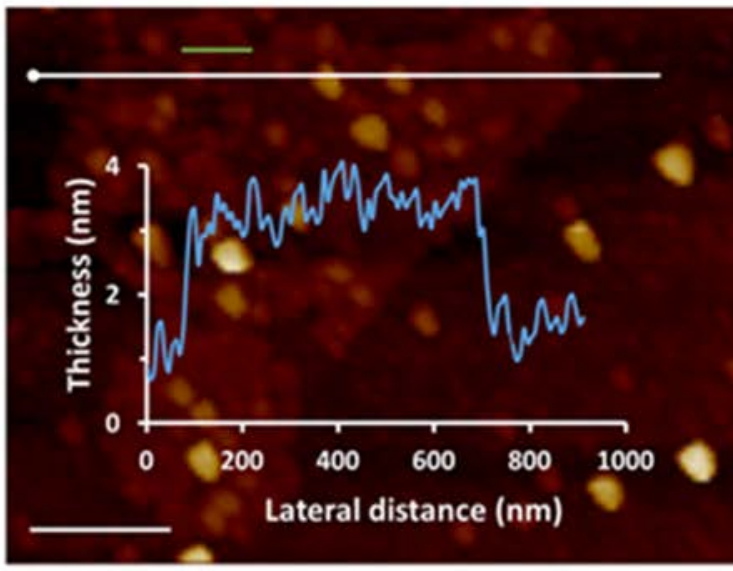

$7 \mathrm{~nm}$

(b)

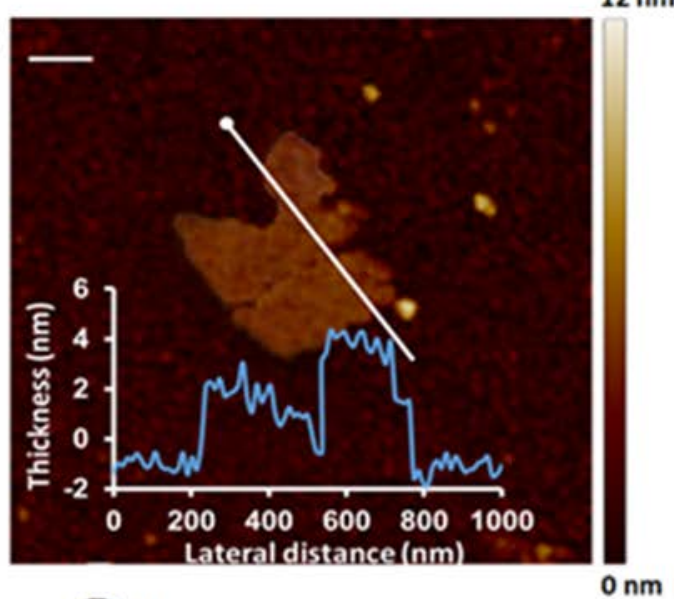

(d)

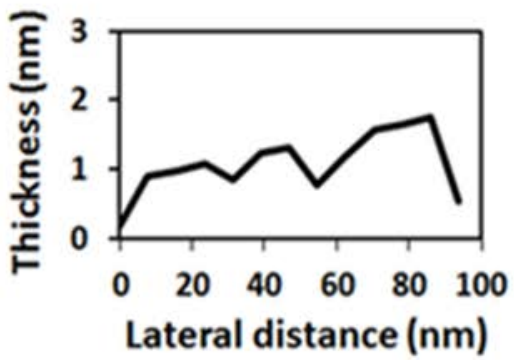

(e)

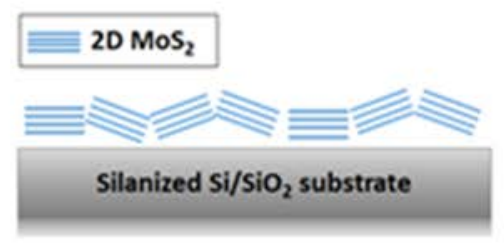

Fig. 2 AFM images and height profiles of 2D MoS 2 nanoflakes synthesised on: (a) APTES, (b) AEAPTMS and (c) DETA silanised Si/SiO substrates. Scale bars represent $200 \mathrm{~nm}$. (d) Height profile along the green line overlaid in image (c). (e) Schematic cross sectional representation of the quasi $2 \mathrm{D} \mathrm{MoS}$ sheet made of adjoining small flakes.

shown in Fig. 2e. It seems that DETA molecules are assembled at different angles on the substrates. Different angles mean various
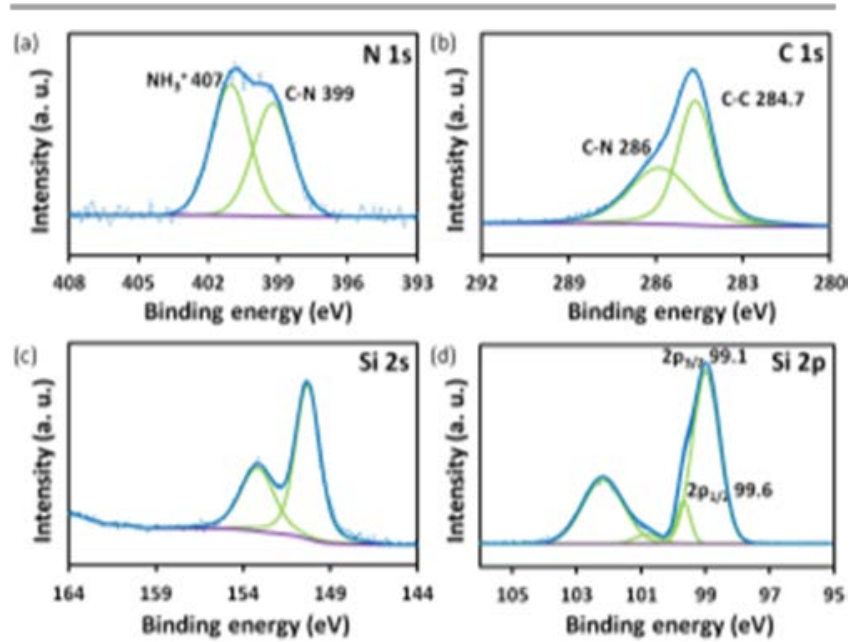

Fig. 3 XPS spectra of (a) N 1s, (b) C 1s, (c) Si 2p, and (d) Si $2 s$ binding energies from the DETA silanised substrate. heights for the $\mathrm{MoS}_{2}$ growth points with reference to the substrate's surface. This, in result, promotes the formation of jagged and adjoining 2D surfaces of $\mathrm{MoS}_{2}$

As in this study DETA produced the largest quasi $2 \mathrm{D} \mathrm{MoS}_{2}$ flakes, the optical and compositional analysis will be more comprehensively presented based on this self-assembled monolayer.

XPS was used for determining the chemical compositions and chemical states of the DETA monolayers and obtained quasi 2D $\mathrm{MoS}_{2}$ sheets. Before we carried out studies on the $\left[\mathrm{MoS}_{4}\right]^{2-}$ anion surface treated samples, as a control experiment, the DETA treated substrates were first investigated by XPS to ensure that all the expected elements of the aminosilane are on the surface. For the DETA silanised substrate, two peaks are seen in the $N$ 1s spectra, one at $399 \mathrm{eV}$ binding energy related to the $\mathrm{C}-\mathrm{N}$ binding and the other at $407 \mathrm{eV}$ due to the protonated amines (Fig. 3a). ${ }^{53,54}$ The deconvoluted $C$ 1s spectrum shows two peaks with binding energy of 284.7 and $286 \mathrm{eV}$, which are ascribed to $\mathrm{C}-\mathrm{C}$ and $\mathrm{C}-\mathrm{N}$ bondings (Fig. $3 b){ }^{53,54} \mathrm{Si} 2 \mathrm{~s}$ has the same peak locations as a previous study (Fig. 3c). ${ }^{54}$ For the spectra of Si $2 p$ peak (Fig. 3d), it shows two binding energies at 99.1 and $99.6 \mathrm{eV}$, corresponding to $\mathrm{Si} 2 \mathrm{p}_{3 / 2}$ and Si $2 p_{1 / 2}$ from the bulk silicon wafer beneath the oxide layer. ${ }^{53}$ The 

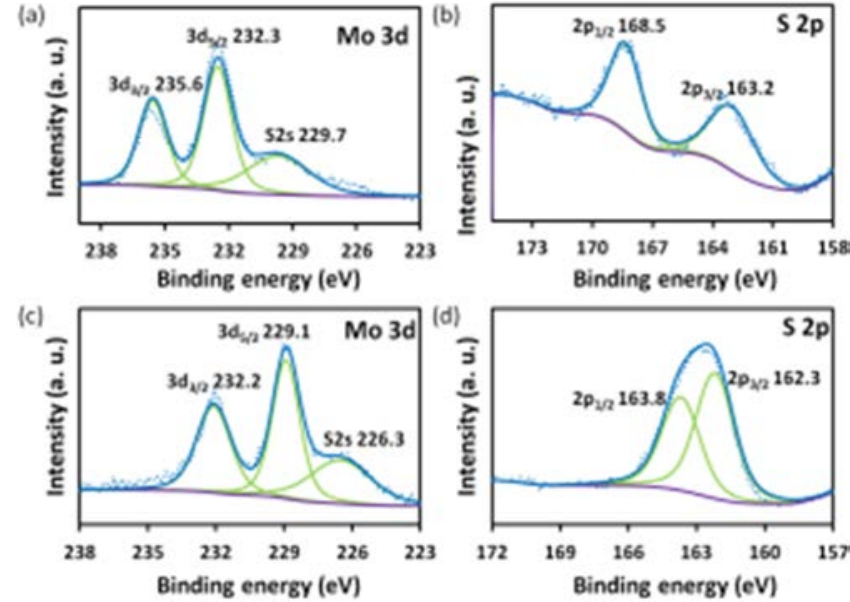

Fig. 4 XPS spectra of (a) Mo $3 d$ and (b) S $2 p$ binding energies from substrate with molybdenum precursor after DETA silanisation. (c) and (d) are the aforementioned elements binding energies of the samples after the combined thermolysis and sulphurisation process.

signal appears at the binding energy of $100.9 \mathrm{eV}$ is contributed by aminosilane ( $\mathrm{Si}-\mathrm{O}$ ) on the silicon dioxide and the one at $102.2 \mathrm{eV}$ is ascribed to silicon dioxide. ${ }^{53}$

For the DETA silanised substrates with the molybdenum precursor, the XPS results for Mo and $S$ binding energies are shown in Fig. 4a and 4b. The deconvoluted Mo 3d spectrum shows that two Mo $3 \mathrm{~d}$ peaks at 232.3 and $235.6 \mathrm{eV}$, corresponding to the $3 \mathrm{~d}_{3 / 2}$ and $3 d_{5 / 2}$ binding energies, respectively, characteristic for the Mo ${ }^{6+}$ state. ${ }^{4}$ The $S 2 s$ peak can be observed at $229.7 \mathrm{eV}$. From S $2 p$ spectra, $S 2 p_{1 / 2}$ and $S 2 p_{3 / 2}$ are located at 168.5 and $163.2 \mathrm{eV}$ (Fig. $4 b)$. The XPS assessments provide good evidence that the DETA silanisation process has been a success.

After the substrates were treated with a combined thermolysis and sulphurisation, obtained quasi $2 \mathrm{D} \mathrm{MoS}_{2}$ flakes were observed to have lower binding energies for Mo $3 d$ and S $2 p$ peaks (Fig. 4c and d). It also exhibited two characteristic Mo 3d peaks at 229.1 and $232.2 \mathrm{eV}$, corresponding to the $3 \mathrm{~d}_{3 / 2}$ and $3 \mathrm{~d}_{5 / 2}$ binding energies for $\mathrm{Mo}^{4+29}$ S $2 \mathrm{~s}$ peak was found at $226.3 \mathrm{eV}$. The sulphur peak for the $2 p$ orbital (Fig. $4 b$ and d) is shifted from 163.2 to $162.3 \mathrm{eV}$ (for $2 p_{3 / 2}$ ) and from 168.5 to $163.8 \mathrm{eV}$ (for $2 p_{1 / 2}$ ). Decreases of binding energy for both Mo and $\mathrm{S}$ elements are due to the change of oxidation state of $\mathrm{Mo}$ from $\mathrm{Mo}^{6+}$ to $\mathrm{Mo}^{4+}$, which leads to a reduction of bonding strength between Mo and S. The XPS results confirm the Mo oxidation transition from +6 to +4 , which means the transition from bonded $\left[\mathrm{MoS}_{4}\right]^{2-}$ anion groups to nearly perfect stoichiometric $\mathrm{MoS}_{2}$ composition after the thermolysis and sulphurisation process.

The XPS results for $\mathrm{MoS}_{2}$ grown on APTES and AEAPTMS silanised substrate were also confirmed to form $\mathrm{MoS}_{2}$. The data are presented in the Supporting Information.

Energy dispersive X-ray (EDX) spectrum measurement was used for characterising the chemical composition of the synthesised $\mathrm{MoS}_{2}$ nanosheets and the result is shown in Figure S3. Peaks associated with Mo and S are found at 2.29 and $2.3 \mathrm{keV}$ in the spectrum. Quantitative analysis shows that the atomic ratio of $\mathrm{S}$ to Mo is approximately 2.1, which further confirms stoichiometric state of $\mathrm{MoS}_{2}$
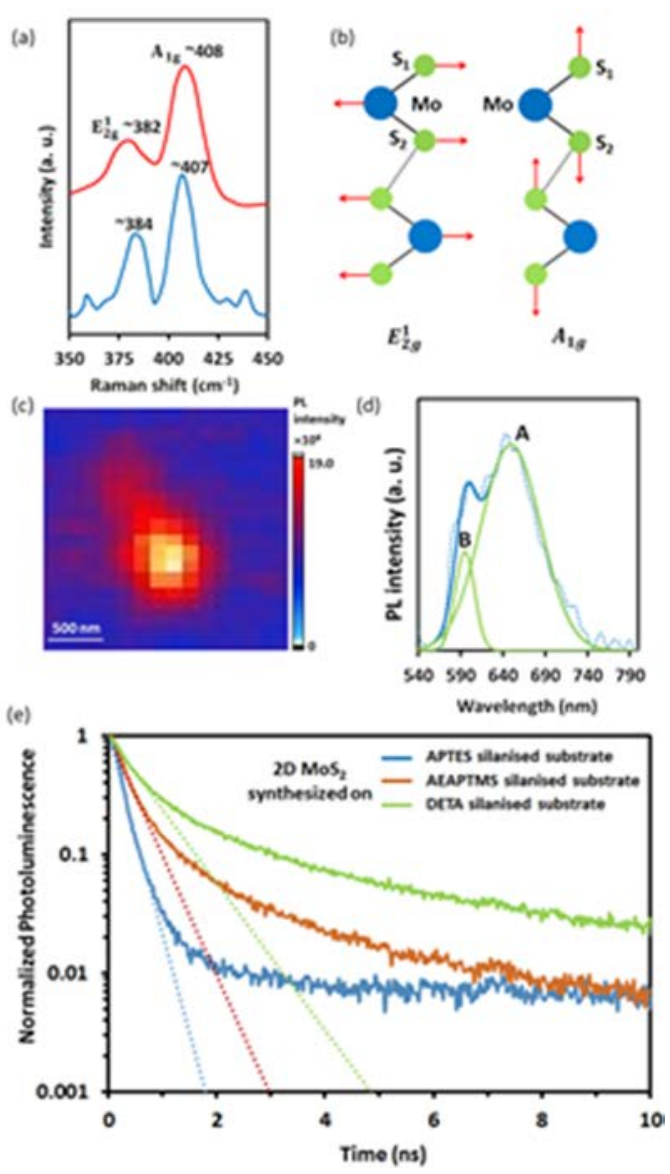

Fig. 5 (a) Raman spectra of DETA salinised samples on the $\mathrm{Si} / \mathrm{SiO}_{2}$ substrate. (b) Schematics of $\mathbf{E}_{\mathbf{2 g}}^{\mathbf{1}}$ and $\mathbf{A}_{\mathbf{1 g}}$ Raman vibrational modes. (c) PL mapping image showing the integrated intensities of $A$ and $B$ exciton peaks. (d) Typical PL spectrum extracted of a sample. (e) PL decay graphs with fitted exponential of three APTES, AEAPTMS and DETA samples for comparison.

Raman spectroscopy is also used for assessing the thickness and number of layers of the obtained quasi $2 \mathrm{D} \mathrm{MoS}_{2}$ flakes on DETA silanised $\mathrm{Si} / \mathrm{SiO}_{2}$ substrate. From Fig. $5 \mathrm{a}$, the spectrum reveals that two characteristic Raman modes of $\mathrm{MoS}_{2}, \mathbf{E}_{2 \mathrm{~g}}^{\mathbf{1}}$ and $\mathbf{A}_{\mathbf{1 g}}$, which represents the in-plane strain and out-of-plane modes (Fig. 5b). The frequency difference between $\mathbf{E}_{\mathbf{2 g}}^{\mathbf{1}}$ and $\mathbf{A}_{\mathbf{1 g}}$ phonons has been shown as an indicator of the number of the layers in $\operatorname{MoS}_{2} \cdot{ }^{55}$ We observe the frequency difference of the $\mathbf{E}_{\mathbf{2 g}}^{\mathbf{1}}$ and $\mathbf{A}_{\mathbf{1 g}}$ peaks in different sample areas to be around 23 to $26 \mathrm{~cm}^{-1}$. The Raman spectra results indicate that 3 to 5 layers can be dominant in different regions of the synthesised flakes. ${ }^{40}$ This Raman spectroscopy results are also consistent with the AFM profiles.

$\mathrm{MoS}_{2}$ nanosheets synthesised in this method have a polycrystalline structure. This can be evidenced in a full range scan of Raman spectroscopy. From Figure S4, Raman spectra at 520 and $300 \mathrm{~cm}^{-1}$ are contributed by $\mathrm{Si} / \mathrm{SiO}_{2}$ substrate. Two $\mathrm{MoS}_{2}$ Raman modes are located at 407 and $384 \mathrm{~cm}^{-1}$. Interestingly, there is an additional peak at $226 \mathrm{~cm}^{-1}$ which is suggested to be a disorderedinduced peak ascribed to $\mathrm{MoS}_{2}$ due to first-order scattering of LA(M) phonons obtained from randomly oriented $\mathrm{MoS}_{2}{ }^{44}$ Besides this, AFM image in Figure 2 of the manuscript also shows that $\mathrm{MoS}_{2}$ sheets are composed of adjoining small nanoflakes with different 
orientations. This can further confirm that the synthesised $\mathrm{MoS}_{2}$ nanosheets in this study have a polycrystalline nature.

$\mathrm{X}$-ray diffraction (XRD) was also conducted for the as grown $\mathrm{MoS}_{2}$ flakes and the result is presented in Figure S5. From the XRD pattern, the typical planar $\mathrm{MoS}_{2}$ peak at $14.5^{\circ}$ which corresponds to (002) plane was not observed, confirming the polycrystalline nature of our quasi $2 \mathrm{D} \mathrm{MoS}_{2}$ sheets.

PL measurements: To investigate the optical properties of the quasi 2D MoS${ }_{2}$ flakes made of adjacent entities in a mosaic formation, PL mapping was performed. PL spectroscopy was also conducted to explore whether the formation of the small flakes cause any improvement in the PL signatures. Fig. $5 \mathrm{c}$ shows the PL intensity-integrated map and the average PL spectrum obtained from the region plotted in Fig. $5 \mathrm{~d}$.

Using the Fityk Software package (version 0.9.2), two Gaussian curves were estimated to fit the obtained PL spectrum. These two peaks are at 590 and $650 \mathrm{~nm}$, which correspond to the energy of the $B$ and $A$ excitons in quasi $2 D M_{2}$. Blue shifting of the PL emission peak was observed compared to the previous report on 2D MoS 2 deposited on glass substrates. ${ }^{56}$ The shift can be due to the combination of two different effects. It may be due to the suppression of $A$ excition in $n$-type doped $\mathrm{MoS}_{2}$ by the $\mathrm{Si} / \mathrm{SiO}_{2}$ substrate, resulting in only $A^{-}$(trion) contributing to $\mathrm{PL}^{57}$ Excitons are generated when photons, with sufficient energy, excite semiconductors like 2D MoS${ }_{2}$. These excitons are electron-hole pairs which are attracted to each other by coulomb forces. These forces become more prominent when the thickness of the thin film decreases. Therefore, nanoflakes with relatively small dimensions contribute to higher energy PL emission. ${ }^{58}$ The shift can also be due to the quantum confinement effect associated to the small dimensions of the adjacent flakes forming the main planes. This observation was also similarly seen in the liquid phase 2D $\mathrm{MoS}_{2}$ with laterally polydispersed samples. ${ }^{19,26}$

To compare the photodynamics of our synthesised quasi 2D $\mathrm{MoS}_{2}$ with previous works, we assess the internal quantum efficiencies of the samples. Internal quantum efficiency $\eta_{i}$ is obtained using the following equations: ${ }^{59}$

$$
\begin{gathered}
\eta_{i}=\frac{\mathrm{Bn}^{2}}{\mathrm{G}} \\
\mathrm{I}_{\mathrm{PL}}=\theta \mathrm{Bn}^{2} \\
\mathrm{G}=\frac{0.4 \times \mathrm{P}_{\text {laser }} \alpha}{\mathrm{A}_{\text {spot }} \mathrm{h} v}
\end{gathered}
$$

Here $\mathrm{B}$ represents the radiative bimolecular coefficient, $\mathrm{n}$ is the photoexcited carrier concentration, and G is the steady state carrier generation/recombination rate, which is calculated using the experimental equation (4). In equation (3), $\theta$ is a constant which can be determined based on the measurement parameters presented in ref 50 and IPL is the integrated PL intensity. In equation (4), Plaser, $\mathrm{A}_{\text {spot, }}$, and $\mathrm{h} v$ are the laser power, excitation area, and excitation photon energy, respectively, and $\boldsymbol{\alpha}$ is $\sim 0.05$ per layer. ${ }^{59}$

Total lateral dimensions of quasi 2D MoS 2 planes sufficiently large to ensure the illumination of the entire nanosheet by the laser for the DETA silanised sample, allowing the determination of the quantum yield. The calculated internal quantum efficiency of our $3 \sim 5$ layered sample is found to be $2.6 \%$, which is possibly indicative of a highly stoichiometry sample. This is associated with theoretical mono layer efficiency of $\sim 11 \%$. Non-radiative recombination processes frequently occur at defect sites and thus the quantum yield can be utilised to assess the degree of stoichiometry. In comparison to our result, high quality mechanically exfoliated monolayer $\mathrm{MoS}_{2}$ flakes feature an intrinsic internal quantum efficiency of $8.3 \%$ at room temperature for one layer. ${ }^{59}$ The highest observed internal quantum efficiency for monolayer $\mathrm{MoS}_{2}$ has been reported to be above $95 \% .{ }^{46}$ To achieve this high efficiency, Amani et. al. designed a process to functionalise mechanically exfoliated flakes using the nonoxidising organic superacid bis(trifluoromethane) sulfonimide. This kind of superacid successfully inhibited defect-mediated nonradiative recombination processes, leading to the largely increased quantum efficiency. ${ }^{46}$ The internal quantum efficiency obtained from ion intercalationassisted exfoliated monolayer $\mathrm{MoS}_{2}$ flakes has been reported to be $1.3 \%,{ }^{60}$ which is less than that of our samples.

The enhancement of internal quantum efficiency may also be due to quantum confinement effects due to the formation of a mosaic of smaller flakes within the structure of quasi 2D MoS 2 . It has been reported the quantum efficiency increases with decreasing of flake sizes, as smaller size of samples lead to fewer nonradioactive recombination. ${ }^{61}$ The idea has been extended to $2 \mathrm{D}$ flakes with small lateral dimensions, generating quantum confinement within thickness and across. ${ }^{19,26}$ As a result, the presence of small flakes may result from an increase of internal quantum efficiency. For our samples, as can be seen from Fig. $2 c$ and d, the synthesised sheets have a mosaic structure, which is composed of adjoining small $\mathrm{MoS}_{2}$ nanoflakes.

$\mathrm{PL}$ radiative decay of quasi $2 \mathrm{D} \mathrm{MoS}$ grown on three different silanised substrates was also measured in this study. Fitting of the data in Figure $5 \mathrm{e}$, the mean excitonic recombination time for quasi 2D MoS 2 grown on APTES, AEAPTMS and DETA were determined to be $0.26,0.43$ and $0.70 \mathrm{~ns}$. Considering that the DETA sample provides the longest recombination time, the assumption that it has the lowest defect and hence a relatively large internal quantum efficiency is confirmed.

All in all, the technique presented in this paper, when further perfected, can be potentially used as a replacement to CVD methods for the deposition of specific configurations of 2D MoS . Advantageously, the as grown layers can also take the shape of patterns on pre-silanised substrates. The method is also compatible with other liquid phase deposition techniques. This method can be transferrable to grow other TMD nanosheets. The reaction in this study is based on the interaction between amine groups of aminosilane and transition metal containing precursor in the form of ammonium salt. TMD nanosheets can be obtained via a combined thermolysis and sulphurisation of the transition metal precursor on silanised $\mathrm{Si} / \mathrm{SiO}_{2}$ substrate.

\section{Materials and Methods}

The $\mathrm{Si}-\mathrm{SiO}_{2}$ wafers $(<100\rangle$, n-doped, 0-100 $\Omega \mathrm{cm}$, $300 \mathrm{~nm}$-thick dry thermal grown silicon dioxide) were purchased from WRS Materials (San Jose, CA, USA). APTES, AEAPTMS and DETA were purchased from Sigma Aldrich. Ammonium tetrathiomolybdate $\left(\left(\mathrm{NH}_{4}\right)_{2} \mathrm{MoS}_{4}\right)$ was ordered from Sigma. Toluene and Dimethylformamide (DMF) was purchased from Sigma. DI water was used throughout the experiment.

\section{Surface functionalisation of $\mathrm{Si} / \mathrm{SiO}_{2}$ substrate with aminosilanes}


The $\mathrm{Si}-\mathrm{SiO}_{2}$ wafers were cut into $10 \times 10 \mathrm{~mm}^{2}$ pieces. The substrates were sequentially cleaned with acetone and 2-propanol in an ultrasonic bath for 3 minutes, respectively, followed by rinsing with DI water and then dried with $\mathrm{N}_{2}$ flow. The substrate $300 \mathrm{~nm} \mathrm{SiO}$ on $\mathrm{Si}\left(\mathrm{SiO}_{2} / \mathrm{Si}\right)$ was first cleaned with a standard piranha solution (a mixture of $3: 1(\mathrm{v} / \mathrm{v}) 30 \% \mathrm{H}_{2} \mathrm{O}_{2}$ and $\left.27 \% \mathrm{NH}_{4} \mathrm{OH}\right)$ at $70{ }^{\circ} \mathrm{C}$ for $30 \mathrm{~min}$. The substrate was functionalised with APTES, AEAPTMS and DETA using the method reported previously. ${ }^{50}$ Briefly, self-assembled monolayers of aminosilanes on $\mathrm{Si} / \mathrm{SiO}_{2}$ substrates with $-\mathrm{NH}_{2}$ functional groups were prepared by soaking the substrates in a $2 \%$ solution of APTES, AEAPTMS and DETA in ethanol for 30 mins. Upon their removal from the solution, the samples were washed thoroughly with ethanol and then blown dry with nitrogen.

\section{Surface attachment of the Mo containing ions on substrates}

$0.15 \mathrm{~g}$ of high purity of $\left(\mathrm{NH}_{4}\right)_{2} \mathrm{MoS}_{4}$ powder was added to $20 \mathrm{~mL}$ of DMF solvent to form a solution. The concentration was chosen based on the suggestion by Liu et al. ${ }^{43}$ The solution was sonicated in a bath sonicator for 10 mins to obtain a homogeneous solution. The silanised substrates were soaked in the aforementioned prepared solution for one hour. This time was chosen as it provided the best coverage on the surface of the substrates, which was inspected under an optical microscope and was based according to the overall change of colour. DFM was used for rinsing before the samples were blown dry with a flow of $\mathrm{N}_{2}$.

\section{Thermolysis process by annealing and sulphurisation}

After soaking, the substrates were immediately moved into a chemical vapour deposition (CVD) furnace in which the sulphur powder was placed in the colder zone at $300^{\circ} \mathrm{C}$ and sample substrates were placed on the reaction zone of the quartz tube and kept at $450^{\circ} \mathrm{C}$. The forming gas was a static gas mixture of $\mathrm{Ar}$ and $\mathrm{H}_{2}(95: 5, \mathrm{vol} / \mathrm{vol})$.

\section{Characterisation of silanised substrate and grown $\mathrm{MoS}_{2}$ thin films}

AFM (Bruker Dimension Icon) in a tapping mode was used for assessing the thickness and topology of the flakes and images were analysed using Scan-assist ${ }^{\circledR}$ Software. Raman spectra were obtained using a confocal microscope system (WITec, alpha 300R) with a $\times 100$ objective (NA $=0.9$ ) in an ambient condition. A $532 \mathrm{~nm}$ laser was used to excite samples which were placed on a piezo crystalcontrolled scanning state. XPS measurements were performed on a Thermo scientific K-Alpha VG-310F instrument using aluminium monochromated X-rays (20 kV, $15 \mathrm{~mA}$ ) with the hemispherical energy analyser set at a pass energy of $20 \mathrm{eV}$ for the peak scans. WCA measurements were conducted using an OCA 20 system (DataPhysics Instruments, GmbH, Germany) with an automatic dispenser. The height of the each drop was confirmed using a CCD camera prior to each measurement to ensure consistency in the drop volume. A $10 \mu \mathrm{L}$ DI water droplet was dropped on the surface of the gel and the angle is measured using the circle fit. The PL mapping was carried out on a custom build instrument using a Quantum GEM $532 \mathrm{~nm}$ and a Fianium WhiteLase (WL-SC400-8 $405 \mathrm{~nm}$ ) laser for excitation delivering $350 \mu \mathrm{W}$ of laser light at the sample position. A Nikon air objective $(100 \times 0.9 \mathrm{NA})$, in combination with a $532 \mathrm{~nm}$ (or $405 \mathrm{~nm}$ ) notch filter, was used.

\section{Conclusions}

In summary, we demonstrated the synthesis of large-area quasi 2D $\mathrm{MoS}_{2}$ nanosheets, made of adjoining flakes, by the aminosilane functionalisation and subsequent thermolysis and sulphurisation of $\mathrm{Si} / \mathrm{SiO}_{2}$ substrates. AFM results show that samples obtained from the DETA functionalisation provided the best foundation to grow relatively large quasi $2 \mathrm{D} \mathrm{MoS}_{2}$ sheets. The characterisation of these thin sheets showed that lateral dimensions in the order of micron or submicron and dominant 3-5 layers were obtained, which are made of nanoflakes with the lateral dimensions in the order of 30 to $50 \mathrm{~nm}$. The number of layers was also confirmed by Raman spectroscopy.

The surface contact angle measurements showed that the lowest wettability belonged to DETA covered substrates. This observation together with the fact that DETA has the longest chain and larger number of amine groups on its chain were associated to the more efficient lateral growth of quasi $2 \mathrm{D} \mathrm{MoS}$ sheets on $\mathrm{SiO}_{2}$ substrate. XPS confirmed the formation of highly stoichiometric 2D $\mathrm{MoS}_{2}$, which was also confirmed by PL decay assessments.

The obtained quasi 2D MoS 2 nanosheets sheets using DETA functionalised substrates had a high internal quantum efficiency and relatively long excitonic recombination time, which were associated to the quantum confinement effect in the mosaic of adjoining small nanoflakes and also the high stoichiometry of the 2D material.

This work presents a new approach for depositing quasi 2D MoS sheet with enhanced optical properties by an intermediate process of silanisation which is particularly compatible with oxide surfaces.

\section{Acknowledgements}

The authors would like to acknowledge the facilities, scientific and technical assistances of the Australian Microscopy and Microanalysis Research Facility (AMMRF) and the Micro Nano Research Facility (MNRF) at RMIT. They also acknowledge help from the Australian Centre for Materials Science and Engineering (CMSE) of the Commonwealth Scientific and Industrial Research Organisation (CSIRO), Australia. The research was financially supported by the Australian Research Council (ARC) through Discovery Project DP140100170. Q. Bao would like to acknowledge the support from ARC DECRA DE120101569 and Discovery Project DP140101501.

\section{References}

37 S. Wu, C. Huang, G. Aivazian, J. S. Ross, D. H. Cobden and X. Xu, ACS Nano, 2013, 7, 2768-2772.

38 A. M. van der Zande, P. Y. Huang, D. A. Chenet, T. C. Berkelbach, Y. You, G.-H. Lee, T. F. Heinz, D. R. Reichman, D. A. Muller and J. C. Hone, Nat. Mater., 2013, 12, 554-561.

39 X. L. Li and Y. D. Li, Chem-Eur. J., 2003, 9, 2726-2731.

40 K.-K. Liu, W. Zhang, Y.-H. Lee, Y.-C. Lin, M.-T. Chang, C.-Y. Su, C.-S. Chang, H. Li, Y. Shi, H. Zhang, C.-S. Lai and L.-J. Li, Nano. Lett., 2012, 12, 1538-1544.

41 J.-M. Yun, Y.-J. Noh, J.-S. Yeo, Y.-J. Go, S.-I. Na, H.-G. Jeong, J. Kim, S. Lee, S.-S. Kim, H. Y. Koo, T.-W. Kim and D.-Y. Kim, J. Mater. Chem. C, 2013, 1, 3777-3783.

42 J. Yang, Y. Gu, E. Lee, H. Lee, S. H. Park, M.-H. Cho, Y. H. Kim, Y.-H. Kim and H. Kim, Nanoscale, 2015, 7, 9311-9319.

43 D. Dumcenco, D. Ovchinnikov, K. Marinov, P. Lazić, M. Gibertini, N. Marzari, O. L. Sanchez, Y.-C. Kung, D. 
Krasnozhon, M.-W. Chen, S. Bertolazzi, P. Gillet, A. Fontcuberta i Morral, A. Radenovic and A. Kis, ACS Nano, 2015, 9, 4611-4620.

44 S. Najmaei, M. Amani, M. L. Chin, Z. Liu, A. G. Birdwell, T. P. O'Regan, P. M. Ajayan, M. Dubey and J. Lou, ACS Nano, 2014, 8, 7930-7937.

45 O. V. Yazyev and Y. P. Chen, Nat. Nanotechnol, 2014, 9, 755767.

46 M. Amani, D.-H. Lien, D. Kiriya, J. Xiao, A. Azcatl, J. Noh, S. R. Madhvapathy, R. Addou, S. KC, M. Dubey, K. Cho, R. M. Wallace, S.-C. Lee, J.-H. He, J. W. Ager, X. Zhang, E. Yablonovitch and A. Javey, Science, 2015, 350, 1065-1068.

47 K. Aslan, I. Gryczynski, J. Malicka, E. Matveeva, J. R. Lakowicz and C. D. Geddes, Curr. Opin. Biotech., 2005, 16, 55-62.

48 R. Tian, O. Seitz, M. Li, W. Hu, Y. J. Chabal and J. Gao, Langmuir, 2010, 26, 4563-4566.

49 A. V. Krasnoslobodtsev and S. N. Smirnov, Langmuir, 2002, 18, 3181-3184.

50 J. A. Howarter and J. P. Youngblood, Langmuir, 2006, 22, 11142-11147.

51 J. Pütz, PhD Thesis, Saarland University, 2000.

52 O. Gonzalez-Ortega and R. Guzman, American J. Anal. Chem., 2014, 5, 932-944.

53 G. Jakša, B. Štefane and J. Kovač, Surf. Interface Anal., 2013, 45, 1709-1713.

54 Y. Wang, M. Lieberman, Q. Hang and G. Bernstein, Int. J. Mol. Sci., 2009, 10, 533-558.

55 H. Li, Q. Zhang, C. C. R. Yap, B. K. Tay, T. H. T. Edwin, A. Olivier and D. Baillargeat, Adv. Funct. Mater., 2012, 22, 13851390.

56 K. P. Dhakal, D. L. Duong, J. Lee, H. Nam, M. Kim, M. Kan, Y. H. Lee and J. Kim, Nanoscale, 2014, 6, 13028-13035.

57 N. Scheuschner, O. Ochedowski, A.-M. Kaulitz, R. Gillen, M Schleberger and J. Maultzsch, Phys. Rev. B, 2014, 89, 125406.

58 G. W. Mudd, S. A. Svatek, T. Ren, A. Patanè, O. Makarovsky, L. Eaves, P. H. Beton, Z. D. Kovalyuk, G. V. Lashkarev, Z. R. Kudrynskyi and A. I. Dmitriev, Adv. Mater., 2013, 25, 57145718.

59 O. Salehzadeh, N. H. Tran, X. Liu, I. Shih and Z. Mi, Nano Lett., 2014, 14, 4125-4130.

60 H. D. Ha, D. J. Han, J. S. Choi, M. Park and T. S. Seo, Small, 2014, 10, 3858-3862.

61 N. F. Borrelli, D. W. Hall, H. J. Holland and D. W. Smith, J. Appl. Phys., 1987, 61, 5399-5409.

1 1. K. Kalantar-zadeh, J. Z. Ou, T. Daeneke, M. S. Strano, M. Pumera and S. L. Gras, Advanced Functional Materials, 2015, 25, 5086-5099.

2 2. M. Chhowalla, H. S. Shin, G. Eda, L.-J. Li, K. P. Loh and H. Zhang, Nat Chem, 2013, 5, 263-275.

3 3. C. Tan and H. Zhang, Chemical Society Reviews, 2015, 44, 2713-2731.

4 4. K. F. Mak, C. Lee, J. Hone, J. Shan and T. F. Heinz, Phys. Rev. Lett., 2010, 105, 136805.

5 5. A. S. George, Z. Mutlu, R. lonescu, R. J. Wu, J. S. Jeong, H. H. Bay, Y. Chai, K. A. Mkhoyan, M. Ozkan and C. S.
Ozkan, Advanced Functional Materials, 2014, 24, 74617466.

6 6. Y.-H. Lee, X.-Q. Zhang, W. Zhang, M.-T. Chang, C.-T. Lin, K.-D. Chang, Y.-C. Yu, J. T.-W. Wang, C.-S. Chang, L.-J. Li and T.-W. Lin, Advanced Materials, 2012, 24, 23202325.

7 7. B. Radisavljevic, A. Radenovic, J. Brivio, V. Giacometti and A. Kis, Nat. Nanotechnol., 2011, 6, 147-150.

8 8. B. Liu, L. Chen, G. Liu, A. N. Abbas, M. Fathi and C. Zhou, ACS Nano, 2014, 8, 5304-5314.

9 9. Y. Che, Y.-C. Lin, P. Kim and C. Zhou, ACS Nano, 2013, 7, $4343-4350$

1010 . H. Li, Z. Yin, Q. He, H. Li, X. Huang, G. Lu, D. W. H. Fam, A. I. Y. Tok, Q. Zhang and H. Zhang, Small, 2012, 8, 6367.

11 11. A. Ambrosi, X. Chia, Z. Sofer and M. Pumera, Electrochemistry Communications, 2015, 54, 36-40.

12 12. M. Z. M. Nasir, Z. Sofer, A. Ambrosi and M. Pumera, Nanoscale, 2015, 7, 3126-3129.

13 13. Y. Wang, B. J. Carey, W. Zhang, A. F. Chrimes, L. Chen, K. Kalantar-zadeh, J. Z. Ou and T. Daeneke, The Journal of Physical Chemistry C, 2016, 120, 2447-2455.

14 14. S. M. Tan and M. Pumera, ACS Applied Materials \& Interfaces, 2016, 8, 3948-3957.

15 15. Z. He and W. Que, Applied Materials Today, 2016, 3, 23-56.

16 16. X. Chia, A. Y. S. Eng, A. Ambrosi, S. M. Tan and M. Pumera, Chemical Reviews, 2015, 115, 11941-11966.

17 17. Q. Weng, X. Wang, X. Wang, C. Zhang, X. Jiang, Y. Bando and D. Golberg, J. Mater. Chem. A, 2015, 3, 3097-3102.

18 18. J.-Z. Wang, L. Lu, M. Lotya, J. N. Coleman, S.-L. Chou, H.-K. Liu, A. I. Minett and J. Chen, Adv. Energy Mater., 2013, 3, 798-805.

19 19. O. Lopez-Sanchez, D. Lembke, M. Kayci, A. Radenovic and A. Kis, Nat. Nanotechnol., 2013, 8, 497-501.

20 20. K. J. Berean, J. Z. Ou, T. Daeneke, B. J. Carey, E. P. Nguyen, Y. Wang, S. P. Russo, R. B. Kaner and K. Kalantar-zadeh, Small, 2015, 11, 5035-5040.

21 21. W. Wu, L. Wang, Y. Li, F. Zhang, L. Lin, S. Niu, D. Chenet, X. Zhang, Y. Hao, T. F. Heinz, J. Hone and Z. L. Wang, Nature, 2014, 514, 470-474.

22 22. J. Z. Ou, A. F. Chrimes, Y. Wang, S. Y. Tang, M. S. Strano and K. Kalantar-zadeh, Nano Letters, 2014, 14, 857863.

23 23. Q. He, Z. Zeng, Z. Yin, H. Li, S. Wu, X. Huang and H. Zhang, Small, 2012, 8, 2994-2999.

24 24. C. Zhu, Z. Zeng, H. Li, F. Li, C. Fan and H. Zhang, J. Am. Chem. Soc., 2013, 135, 5998-6001.

25 25. K. Kalantar-zadeh and J. Z. Ou, ACS Sensors, 2015, DOI: 10.1021/acssensors.5b00142.

26 26. A. H. Loo, A. Bonanni, A. Ambrosi and M. Pumera, Nanoscale, 2014, 6, 11971-11975.

27 27. S. Wi, H. Kim, M. Chen, H. Nam, L. J. Guo, E. Meyhofer and X. Liang, ACS Nano, 2014, 8, 5270-5281.

2828 . J. N. Coleman, M. Lotya, A. O'Neill, S. D. Bergin, P. J. King, U. Khan, K. Young, A. Gaucher, S. De, R. J. Smith, I. 
V. Shvets, S. K. Arora, G. Stanton, H.-Y. Kim, K. Lee, G. T. Kim, G. S. Duesberg, T. Hallam, J. J. Boland, J. J. Wang, J. F. Donegan, J. C. Grunlan, G. Moriarty, A. Shmeliov, R. J. Nicholls, J. M. Perkins, E. M. Grieveson, K. Theuwissen, D. W. McComb, P. D. Nellist and V. Nicolosi, Science, 2011, 331, 568-571.

29 29. Y. Wang, J. Z. Ou, S. Balendhran, A. F. Chrimes, M. Mortazavi, D. D. Yao, M. R. Field, K. Latham, V. Bansal, J. R. Friend, S. Zhuiykov, N. V. Medhekar, M. S. Strano and K. Kalantar-zadeh, ACS Nano, 2013, 7, 1008310093.

30 30. Y. Wang, J. Z. Ou, A. F. Chrimes, B. J. Carey, T. Daeneke, M. M. Y. A. Alsaif, M. Mortazavi, S. Zhuiykov, N. Medhekar, M. Bhaskaran, J. R. Friend, M. S. Strano and K. Kalantar-Zadeh, Nano Letters, 2015, 15, 883-890.

31 31. E. P. Nguyen, B. J. Carey, J. Z. Ou, J. van Embden, E. D. Gaspera, A. F. Chrimes, M. J. S. Spencer, S. Zhuiykov, K. Kalantar-zadeh and T. Daeneke, Advanced Materials, 2015, 27, 6225-6229.

32 32. G. Eda, H. Yamaguchi, D. Voiry, T. Fujita, M. Chen and M. Chhowalla, Nano Lett., 2011, 11, 5111-5116.

33 33. F. Bonaccorso, A. Lombardo, T. Hasan, Z. Sun, L. Colombo and A. C. Ferrari, Materials Today, 2012, 15, 564-589.

34 34. Y. Zhan, Z. Liu, S. Najmaei, P. M. Ajayan and J. Lou, Small, 2012, 8, 966-971.

35 35. X. Wang, H. Feng, Y. Wu and L. Jiao, Journal of the American Chemical Society, 2013, 135, 5304-5307.

36 36. S. Balendhran, J. Z. Ou, M. Bhaskaran, S. Sriram, S. Ippolito, Z. Vasic, E. Kats, S. Bhargava, S. Zhuiykov and K. Kalantar-zadeh, Nanoscale, 2012, 4, 461-466.

37 37. S. Najmaei, Z. Liu, W. Zhou, X. Zou, G. Shi, S. Lei, B. I. Yakobson, J.-C. Idrobo, P. M. Ajayan and J. Lou, Nat Mater, 2013, 12, 754-759.

38 38. C.-C. Huang, F. Al-Saab, Y. Wang, J.-Y. Ou, J. C. Walker, S. Wang, B. Gholipour, R. E. Simpson and D. W. Hewak, Nanoscale, 2014, 6, 12792-12797.

39 39. Y. Yu, C. Li, Y. Liu, L. Su, Y. Zhang and L. Cao, Scientific Reports, 2013, 3, 1866.

40 40. S. Wu, C. Huang, G. Aivazian, J. S. Ross, D. H. Cobden and X. Xu, ACS Nano, 2013, 7, 2768-2772.

41 41. A. M. van der Zande, P. Y. Huang, D. A. Chenet, T. C. Berkelbach, Y. You, G.-H. Lee, T. F. Heinz, D. R. Reichman, D. A. Muller and J. C. Hone, Nat Mater, 2013, 12, 554-561.

42 42. X. L. Li and Y. D. Li, Chemistry - A European Journal, 2003, 9, 2726-2731.

43 43. K.-K. Liu, W. Zhang, Y.-H. Lee, Y.-C. Lin, M.-T. Chang, C.Y. Su, C.-S. Chang, H. Li, Y. Shi, H. Zhang, C.-S. Lai and L.J. Li, Nano. Lett., 2012, 12, 1538-1544.

44 44. G. L. Frey, R. Tenne, M. J. Matthews, M. S. Dresselhaus and G. Dresselhaus, Physical Review B, 1999, 60, 28832892.

45 